\title{
¿SENDEROS DIVERGENTES Y RESULTADOS \\ CONVERGENTES? LA DESIGUALDAD \\ ECONÓMICA EN EUROPA Y AMÉRICA LATINA \\ EN LAS PRIMERAS DÉCADAS DEL SIGLO XXI
}

DIVERGENT PATHS AND CONVERGENT RESULTS?

ECONOMIC INEQUALITY IN EUROPE AND LATIN

AMERICA IN THE FIRST DECADES OF THE $21^{\text {ST }}$ CENTURY

\section{DES CHEMINS QUI DIVERGENT POUR DES RÉSULTATS QUI CONVERGENT? INÉGALITÉS ÉCONOMIQUES EN EUROPE ET EN AMÉRIQUE LATINE AU COURS DES PREMIÈRES DÉCENNIES DU XXIème SIÈCLE}

\author{
Agustín Salvia \\ Instituto Gino Germani, CoNICET y \\ Universidad Católica Argentina, \\ Observatorio de la Deuda Social \\ Argentina \\ agustin_salvia@uca.edu.ar \\ SAntiago Poy \\ Universidad Católica Argentina, \\ ConicET, Observatorio de la Deuda \\ Social Argentina \\ santiago_poy@uca.edu.ar \\ Guido LAMARMORA \\ Universidad Católica Argentina, \\ Observatorio de la Deuda Social \\ Argentina \\ glamarmora@uca.edu.ar
}

Resumen: El artículo analiza los cambios en la desigualdad económica intra e interregional en América Latina y Europa. Sobre una muestra de 44 países, se examina el grado en que los factores económico-productivos junto con las políticas de bienestar configuran diferencias estructurales en materia de desigualdad entre regiones y en el interior de éstas en el periodo 2000-2017. Los 
resultados revelan que se redujo la brecha de desigualdad interregional como resultado de dinámicas intrarregionales diferentes: la desigualdad disminuyó en la mayor parte de los países latinoamericanos y creció en los europeos. El análisis de las trayectorias nacionales muestra que ello se debió a cambios simultáneos en el estilo de crecimiento y en la eficiencia redistributiva del gasto social.

Palabras clave: desigualdad distributiva; políticas sociales; trayectorias nacionales; desigualdades regionales.

ABSTRACT: The article analyzes the changes in intra- and inter-regional economic inequality in Latin America and Europe. Taking a sample of 44 countries, it examines the extent to which economic and productive factors together with welfare policies bring about structural differences in terms of inequality between and within regions over the period 2000-2017. The results reveal that the inter-regional inequality gap was reduced as a result of different intra-regional dynamics: inequality fell in most Latin American countries while it rose in European countries. The analysis of national trajectories shows that this was due to simultaneous changes in the style of growth and in the redistributive efficiency of social spending.

Keywords: distributive inequality; social policies; national trajectories; regional inequalities.

\section{Traducción de Fionn Petch, CM Idiomas}

Résumé: Cet article se penche sur l'évolution des inégalités économiques intra et interrégionales, en Amérique latine et en Europe. Sur la base d'un échantillon de 44 pays, on y évalue dans quelle mesure les facteurs économiques et productifs ainsi que les politiques sociales ont déterminé des différences structurelles en matière d'inégalité entre et à l'intérieur des régions, durant la période 2000-2017. Nos résultats démontrent que des dynamiques différentes à l'intérieur des régions ont eu pour conséquence une réduction des inégalités entre celles-ci: dans la majorité des pays latino-américains, les écarts se sont réduits, tandis qu'ils s'amplifiaient au sein des pays européens. Comme le démontre l'analyse du parcours suivi par les différents pays, ceci est dû à une évolution simultanée des modes de croissance et de l'efficience du rôle redistributif joué par les dépenses sociales. 
Mots clés: inégalité en matière distributive; politiques sociales; parcours nationaux; inégalités régionales.

Traducción de ARIEL Elbaz, CM IDIOMAS

Fecha de recepción: marzo de 2020

Fecha de aceptación: octubre de 2020

\section{INTRODUCGIÓN}

$\tau$

a comunidad internacional ha logrado grandes avances en la superación de la pobreza. En las últimas tres décadas, casi mil millones de personas abandonaron la pobreza extrema a escala global. ${ }^{1}$ Más allá de estos progresos, persisten desigualdades y grandes disparidades en la distribución del ingreso que parecen afectar las capacidades de desarrollo económico e integración social. ${ }^{2}$ Existe creciente consenso acerca de los desafíos que representa la desigualdad distributiva para el funcionamiento y la estabilidad de los sistemas políticos democráticos. ${ }^{3}$

El objetivo de este artículo es analizar los cambios en la desigualdad de la distribución del ingreso familiar en América Latina y Europa durante el periodo 2000-2017, atendiendo

${ }^{1}$ Según datos del Banco Mundial a partir de una línea de 1.90 USD diarios, con paridad de poder adquisitivo (PPA). Véase The World Bank, Understanding Poverty, Open Data (sitio de internet), https://www.world bank.org/en/understanding-poverty

${ }^{2}$ Organización de las Naciones Unidas, ONU, Transformar nuestro mundo. Agenda 2030 para el desarrollo sostenible, Ginebra, onu, 2013; Organización para la Cooperación y el Desarrollo Económico, oCDE, Perspectivas económicas de América Latina y el Caribe 2014, Logística y competividad para el desarrollo, OCDE, 2013; The World Bank, Globalization, Poverty, and Inequality since 1980, Oxford, University Press, 2005.

${ }^{3}$ B. Milanovic, Desigualdad mundial. Un nuevo enfoque para la era de la globalización, México, FCE, 2016; G. Therborn, La desigualdad mata, Madrid, Alianza Editorial, 2013. 
a las diferencias estructurales que caracterizan a las matrices distributivas de cada región. A partir de una estrategia analítica comparativa entre regiones y en el interior de ellas, el artículo examina el grado en que los cambios en la desigualdad pueden derivarse de patrones explicativos disímiles. En particular, se busca modelar el papel de la "distribución primaria" y "secundaria"4 de ingresos en la estructuración de las desigualdades en cada país, entre países de cada región y entre ambas regiones, con el fin de comprender su relevancia en los procesos de convergencia y divergencia en las trayectorias nacionales y regionales observadas. En esta línea, dos interrogantes orientaron la indagación: ¿en qué medida los factores económico-productivos -asociados a la distribución primaria del ingreso- y los relacionados con las políticas sociales o de bienestar -que remiten a la distribución secundaria- organizan patrones distributivos estructuralmente diferentes entre América Latina y Europa? ¿Qué papel han desempeñado tales factores en los cambios de ciclo distributivo y en la trayectoria seguida por la desigualdad en las distintas regiones? ${ }^{5}$

La desigualdad distributiva en numerosos países del mundo -en especial, en los más desarrollados- se ha incrementado durante las últimas décadas. ${ }^{6}$ Este resultado contradice la teoría más popular sobre el comportamiento de la distri-

${ }^{4}$ La literatura económica suele distinguir las fuentes de distribución "primaria" del ingreso (distribución de ingresos en la relación capitaltrabajo) y las fuentes de distribución "secundaria" (transferencias públicas o privadas surgidas de rentas, regalos o impuestos), cuyo origen es, de alguna manera, el valor agregado generado por la relación capital-trabajo o el saldo comercial del país. P. Krugman, P. Wells y M. Olney, Fundamentos de economía, Buenos Aires, Reverté, 2008.

${ }^{5} \mathrm{Si}$ bien la desigualdad económica se refiere en general a la distribución del ingreso entre individuos de un país, también incluye la desigualdad en el nivel de territorios o regiones. Así, es posible afirmar que Europa es la región con mayor equidad distributiva del planeta y que América Latina es una de las más desiguales. World Inequality Database, World Inequality Lab, Informe sobre la desigualdad global 2018, 2018, https:/ / wir2018. wid.world

${ }^{6} \mathrm{ONU}$, op. cit. 
bución del ingreso en el largo plazo -propuesta por Kuznets en los cincuenta- ${ }^{7}$ de acuerdo con la cual, ante mayores niveles de desarrollo cabía esperar una tendencia decreciente de la desigualdad. Por ello, distintos investigadores han ligado la tendencia reciente con un cambio en la participación de las familias e individuos más ricos en la distribución del ingreso. Su principal conclusión es que tal participación adoptó forma de $U$ : disminuyó durante la primera mitad del siglo xx y desde entonces se incrementó. ${ }^{8}$ Piketty deriva este comportamiento del aumento de la tasa de retorno del capital por encima del ritmo de crecimiento económico, que condujo a la concentración de la riqueza, y Milanovic propone el concepto de "ciclos de Kuznets", en el que fuerzas de distinto sentido e intensidad (como el cambio tecnológico, la expansión educativa o los sistemas tributarios) determinan aumentos y decrementos de la desigualdad. ${ }^{9}$

Por otra parte, la desigualdad entre países y regiones refleja el efecto combinado de la desigualdad en cada país y entre ellos. Algunos estudios pioneros sugerían que luego de un primer periodo de aumento de las brechas entre países, se registraba una reducción de la desigualdad dentro de los países, pero un aumento de la brecha entre éstos según sus niveles de ingreso medio. Más recientemente la tendencia sería otra: la desigualdad tiende a aumentar en los países y se reduce entre ellos. Esta forma de $U$ invertida en la desigualdad entre países se presenta usualmente como base de optimismo que asegura procesos de convergencia entre regiones. Sin embargo, si-

7 S. Kuznets, "Economic Growth and Income Inequality", American Economic Review, 45, 1955, pp. 1-28.

${ }^{8}$ A. Atkinson, Desigualdad: ¿Qué podemos hacer?, México, Fondo de Cultura Económica, 2016; A. Atkinson, T. Piketty y E. Sáez, “Top Incomes in the Long Run of History", Journal of Economic Literature, 49, núm. 1 (2011), pp. 3-71; J. Stiglitz. "Capital Market Liberalization, Economic Growth, and Instability”, World Development, 28, núm. 6 (2000), pp.1075-1086.

9 T. Piketty, El capital en el siglo XXI. Madrid: FCE, 2014; B. Milanovic. Desigualdad mundial. Un nuevo enfoque para la era de la globalización, México DF: FCE, 2016. 
guiendo a Atkinson, hay razones para ser cautelosos. ${ }^{10} \mathrm{En}$ primer lugar, mientras las brechas se reducen, las diferencias absolutas en cuanto a ingresos per cápita reales continúan ensanchándose. En segundo lugar, si bien algunos grandes países han crecido de manera veloz (como China o India), otros países en desarrollo lo han hecho a tasas más bajas.

Desde un enfoque comparativo, este artículo se propone sistematizar un esquema analítico que considera las diferentes formas en las que el modelo de crecimiento económico y la productividad laboral (distribución primaria del ingreso) y las políticas sociales (distribución secundaria) estructuran la desigualdad del ingreso familiar. Se considera particularmente relevante evaluar de qué manera y en qué sentido los diferentes senderos transitados, más allá del punto de partida o de llegada de cada país en materia de equidad distributiva, encuentran explicación en la forma de articulación de dichos vectores. Al respecto, cabe señalar que las diferencias en materia de productividad y desarrollo entre Europa y América Latina son ostensibles y ampliamente reconocidas. Además, mientras que las políticas sociales implementadas por los Estados de bienestar europeos han posibilitado acumular altos grados de productividad, prosperidad económica y cohesión social, los sistemas de seguridad social latinoamericanos han sido tradicionalmente estratificados, regresivos y de baja densidad. ${ }^{11}$ En este punto, mientras que el papel de los

${ }^{10}$ Atkinson, 2016, op. cit., pp. 72-73.

${ }^{11}$ A. M. Guillén, D. Luque y S. González, "El Modelo Social Europeo: evolución y retos", en E. del Pino y J. Rubio (eds.), Los Estados del bienestar en la encrucijada. Políticas sociales en perspectiva comparada, Madrid, Tecnos, 2016; L. Moreno y B. Palier, "The Europeanization of Welfare: Paradigm shifts and social policy reforms", en P. Taylor-Gooby (ed.), Ideas and Welfare State Reform in Western Europe, Nueva York, Palgrave Macmillan, 2016, pp. 145-175; C. Barba Solano, "América Latina: regímenes de bienestar en transición durante los años noventa", Caderno CRH, 20, núm. 50 (2007), pp. 197-211; F. Filgueira, "Modelos de desarrollo, matriz del Estado social y herramientas de las políticas sociales latinoamericanas", en S. Cecchini, F. Filgueira, R. Martínez y C. Rossel (eds.), Instrumentos de protección social. Caminos latinoamericanos hacia la universalización, Santiago de Chile, Cepal, 2015, pp. 49-84. 
mecanismos de mercado, tecnológicos y productivos, ha sido abordado de forma recurrente por la literatura, el modo en que tales procesos se articulan con la distribución secundaria en la configuración de trayectorias de desigualdad disímiles ha sido menos atendido.

A modo de hipótesis de trabajo, se argumenta que los comportamientos "convergentes" en materia de equidad distributiva entre países y regiones que se caracterizan por diferencias estructurales de desarrollo económico y de modelos de intervención social encuentran una más precisa explicación cuando se los relaciona con la evolución de la productividad y del gasto social, de manera que la tendencia observada resultaría de lógicas de estructuración divergentes. Para evaluar esta proposición, el artículo analiza, en primer lugar, qué tan diferentes son las sociedades de ambas regiones en materia de PIB per cápita, pobreza y desigualdad distributiva, así como los senderos de desarrollo transitados por ambas regiones durante las últimas décadas. En segundo lugar, asumiendo la presencia de matrices de bienestar-inequidad desiguales entre regiones-países, el estudio procura descifrar los cambios distributivos ocurridos durante las últimas décadas: para ello, se ponen en correspondencia la productividad del trabajo, el gasto social y sus efectos sobre la desigualdad para cada país. Por último, se ajustó un modelo factorial de componentes principales, a través del cual se analizaron las trayectorias de cada país en torno a ejes estructurales que explican el comportamiento de la desigualdad distributiva.

\section{Antecedentes}

En los últimos años se ha verificado un creciente interés académico por la desigualdad económica, por la concentración de la riqueza en grupos cada vez más reducidos de personas, familias y corporaciones y por las implicaciones políticas de estos procesos sobre el funcionamiento de las sociedades 
democráticas. ${ }^{12}$ El estudio de las desigualdades económicas a nivel mundial se centra en la distribución del ingreso. Desde esta perspectiva, se asume que la renta corriente de los hogares constituye un factor central para el bienestar de la población.

La distribución del ingreso en de cada país resulta de la compleja interacción de factores económicos, demográficos, sociales e institucionales. En las economías capitalistas, el nivel de renta está relacionado con la productividad y, por lo tanto, depende del grado de tecnología y del capital físico utilizado. Las evidencias sugieren que los incrementos de productividad están acompañados por una mejora en las condiciones de vida en tanto operen instituciones laborales, sistemas tributarios y de seguridad social que favorezcan la redistribución. En efecto, existe un consenso creciente acerca de que el crecimiento económico no basta para reducir la pobreza si no es inclusivo o equitativo. ${ }^{13}$ A menudo, en los países más desiguales suelen predominar los sindicatos débiles, la flexibilidad laboral, los sistemas tributarios regresivos y los esquemas de seguridad social de reducido alcance. ${ }^{14}$ En este sentido, si bien la desigualdad económica está modelada de forma prioritaria por la distribución primaria de la renta generada en la relación capital-trabajo, las transferencias públicas o privadas de ingresos -sean a través de la seguridad social o las estrategias sociofamiliares-cumplen un papel relevante en el modo en que se reducen, compensan o agravan las desigualdades económicas que resultan de la distribución primaria. ${ }^{15}$ Estos

${ }^{12}$ Véase una síntesis en A. Atkinson, 2016, op. cit..

${ }^{13}$ B. Keeley, Income Inequality: The Gap Between Rich and Poor. OECD Insights, París, oCDE, 2015; Organización para la Cooperación y el Desarrollo Económico, ocDE, Perspectivas Económicas de América Latina y el Caribe 2014. Logística y Competividad para el Desarrollo, OCDE, 2013.

${ }^{14}$ Organización Internacional del Trabajo, oIT, Wage-led Growth: An equitable strategy for economic recovery, Ginebra, OIT, 2014.

${ }^{15}$ Es posible distinguir dos tipos de instituciones reguladoras de la desigualdad: las predistributivas, que actúan ex ante sobre el origen de las desigualdades en el mercado de trabajo, y las posdistributivas, que actúan 
mecanismos de distribución secundaria son crecientemente importantes en los países en desarrollo, a partir de la generalización de las políticas de asistencia social de poblaciones desfavorecidas. ${ }^{16}$

Existe consenso en cuanto a que el periodo central del siglo xx estuvo marcado por una reducción de la desigualdad distributiva de la renta global en la mayor parte de los países del mundo. La evolución reciente, en cambio, ha quedado bien representada por las contribuciones de Piketty, quien mostró el aumento de la concentración de la riqueza desde $1980 .{ }^{17} \mathrm{Si}$ bien hay coincidencia en cuanto a que las tendencias predominantes en el siglo xx se habrían revertido en las últimas décadas -registrándose un nuevo aumento en la brecha de ingresos entre pobres y ricos-, la literatura no es concluyente en cuanto a la tendencia que ha seguido la distribución de la renta entre países y regiones del mundo, ni en cuanto a los factores que podrían explicar los diferentes procesos.

Por una parte, la evidencia parece confirmar un ciclo de relativa convergencia entre regiones durante la primera década y media del siglo xxi. Por otra, los trabajos que han estudiado la relación general existente entre la desigualdad, el crecimiento y la pobreza tampoco tienen una respuesta definitiva en cuanto a cómo y en qué medida la primera favorece u obstaculiza a los segundos. ${ }^{18}$ Sin embargo, los argumentos tienden a coincidir en las limitaciones tanto teóricas como

ex post para mitigar las desigualdades cuyo origen se encuentra en el mercado laboral. Véase H. Zalakain y B. Barragué, Repensar las políticas sociales: predistribución e inversión social, Madrid, Editorial Grupo 5, 2017.

16 A. Barrientos y D. Hulme, "Social protection for the poor and poorest: An introduction”, en A. Barrientos y D. Hulme (eds.), Social protection for the poor and poorest, Londres, Palgrave Macmillan, 2008, pp. 3-24.

17 T. Piketty, op. cit., 2014.

18 A. Atkinson, 2016, op. cit.; B. Keeley, Income Inequality: The Gap Between Rich and Poor. OECD Insights,. París, OCDE, 2015; B. Milanovic, op. cit.; R. Molero-Simarro, "Growth and inequality revisited: the role of primary distribution of income. A new approach for understanding today's economic and social crises", Cambridge Journal of Economics, 41, núm. 2 (2016), pp. 367-390. 
empíricas del enfoque de Kuznets en lo que respecta a la contribución causal del crecimiento como factor posterior de igualación social. El aparente fracaso de Kuznets refleja en realidad otro problema que enfrentan los economistas al determinar el vínculo entre desigualdad y crecimiento, a saber: que si existe una relación, no parece ser directa.

Tanto la investigación empírica como los principales organismos económicos mundiales coinciden en señalar que la excesiva desigualdad no es buena para el crecimiento. En particular, según la Organización para la Cooperación y el Desarrollo Económicos, oCDE, una mayor brecha de riqueza lleva a que las familias de bajos ingresos inviertan menos en educación y formación en competencias, lo cual afecta el crecimiento al reducir la oferta de trabajadores calificados. Otros autores ponen bajo consideración el efecto de la desigualdad sobre la inestabilidad política y social y sobre los procesos de colusión que ligan a los sectores políticos con las clases ricas, logrando que éstas últimas se apropien de una parte mayor de los excedentes económicos, con lo que afectan la demanda de bienes de consumo y la distribución entre las clases más pobres. ${ }^{19}$

Pero más allá de preguntarse si la desigualdad afecta el crecimiento, no menos importante es examinar de qué modo el nivel de productividad económica de los países determina, condiciona o influye -por diferentes vías- en el sentido de la distribución tanto primaria como secundaria del ingreso. La distribución primaria de la renta tiene lugar en el proceso de producción y está determinada por el modo en que el valor agregado se distribuye en el mercado laboral entre salarios y capital, incluyendo los ingresos mixtos del trabajador o profesional autónomo. Para cambiar la distribución primaria hay que modificar la productividad marginal y las regulaciones laborales, lo cual impacta en los salarios y las ganancias.

${ }^{19} \mathrm{~J}$. Ostry y A. Berg, Inequality and Unsustainable Growth: Two Sides of the Same Coin?, Washington DC, International Monetary Fund, 2011; Stiglitz, op. cit. 
Por su parte, la distribución secundaria del ingreso es la que se realiza a través la renta o las transferencias, sean públicas o privadas. Está centrada principalmente en las transferencias de los gobiernos a las familias a través de los sistemas -contributivos o no-de seguridad o protección social, y en los mecanismos públicos o privados de distribución de rentas, regalías, préstamos o regalos.

En el marco de los estudios nacionales, la recopilación y análisis de estadísticas ha permitido constatar una relación creciente -según el modelo político-económico- entre el desarrollo productivo y el incremento del porcentaje del ingreso concentrado en los cuantiles de renta más altos. Sin embargo, los efectos finales sobre la desigualdad dependen del modo en que los gobiernos administran y distribuyen los recursos generados por el mayor crecimiento o, incluso, el modo en que logran ser pautadas las relaciones capital-trabajo y las remuneraciones mínimas asociadas a cada mercado. Es decir, la productividad del trabajo y sus diferentes fuentes, por una parte, y el efecto redistributivo del gasto social, por otra, son factores estructurales centrales que intervienen en las matrices nacionales y regionales de distribución del ingreso. ${ }^{20}$

Durante el último cuarto del siglo xx y el actual siglo xxI, tanto Europa occidental como América Latina han experimentado fuertes transformaciones en sus estructuras sociales

${ }^{20}$ Los estudios que abordan la correlación entre capacidades productivas y distribución interpersonal de la renta establecen relaciones puramente empíricas entre ellas. M. Adler y K. Schmid, Factor Shares and Income Inequality: Empirical Evidence from Germany 2002-2008, IAw Discussion papers, núm. 82, mayo de 2012, http:/ /www.iaw.edu/RePEc/iaw/pdf/iaw_ dp_82.pdf; E. Daudey y C. García-Peñalosa, "The personal and the factor distributions of income in a cross- section of countries", The Journal of Development Studies 43, núm. 2 (2007), pp. 812-829. Algunas excepciones son A. Atkinson, The economics of inequality, Oxford, University Press, 1975; y A. Glyn, Functional Distribution and Inequality, Oxford, University Press, 2009. De hecho, Glyn concluye que hay "necesidad de una mayor investigación para descubrir las causas de las oscilaciones sufridas por las participaciones factoriales y establecer una conexión entre la distribuciones funcional y personal de la renta" (p. 104). 
en el contexto de los cambios globales sufridos por las condiciones político-económicas internacionales, con alcance e impacto diferentes dependiendo del modo en que cada sociedad pudo absorber, regular y procesar tales tendencias. El siglo XXI es una etapa de agotamiento, transición y redefinición de estos procesos. El impacto de estos cambios sobre los patrones de reproducción social de las desigualdades económicas, en sentido y magnitud, no ha sido suficientemente abordado por la literatura. Si bien hay estudios de casos nacionales o basados en comparaciones entre países de una misma región, son escasos los abordajes interregionales que profundicen los factores sociales subyacentes a estas desigualdades.

Durante las últimas décadas, América Latina experimentó numerosas transformaciones estructurales con impactos sobre la distribución del ingreso. Se pasó de un modelo de desarrollo orientado al mercado interno hacia otro centrado en las exportaciones, las reformas estructurales promercado y las privatizaciones de empresas públicas. Estas modificaciones promovieron, en general, el aumento de la desigualdad distributiva entre los setenta y los noventa. En contraste, durante los 2000, el crecimiento del ingreso medio favoreció la retracción de la pobreza y las políticas redistributivas (en particular, la expansión de los sistemas de asistencia social y pensiones) estimularon la reducción de la desigualdad. ${ }^{21}$

Por su parte, Europa estuvo sometida a un doble efecto de desigualdad. Si bien el proceso de integración económica europea disminuyó las desigualdades entre las naciones hasta la primera década del siglo xxi, la convergencia se interrumpió parcialmente debido a los efectos de la crisis de 2008. Las políticas de ajuste afectaron las partidas presupuestarias sociales y de bienestar, lo que impactó en las condiciones de vida y provocó un aumento de la pobreza (especialmente en los países del sur de Europa). Por lo tanto, existe en la Unión Europea una desigualdad significativa de base territorial. Sin embargo, el mantenimiento más o menos generalizado de

${ }^{21}$ Barba Solano, art. cit.; Filgueira, art. cit. 
un modelo extendido de bienestar, ampliamente legitimado por los sectores políticos y sociales, parece poner límites al deterioro en el contexto de la Gran Recesión. ${ }^{22}$

En este marco, el presente trabajo ofrece evidencias que permiten descifrar la naturaleza de los cambios recientes operados en la desigualdad económica entre y en el interior de Europa y América Latina. Esta tarea se efectúa mostrando el diferente papel distributivo que desempeñan, según sea el caso, las fuentes de ingreso, como expresión del distinto papel que pueden ejercer la productividad laboral, las remuneraciones y las políticas sociales.

\section{Estudio Comparado de PAÍsES de AmÉRICA LATINA Y EUROPA}

La comparación de las estructuras socioeconómicas europeas y latinoamericanas permite advertir diferencias sistemáticas. En este apartado se ofrecen evidencias sobre las diferentes condiciones regionales que modelan las dinámicas de acumulación, intervención social del Estado y distribución del ingreso para una muestra amplia y diversa de sistemas nacionales. La investigación se sustentó en fuentes estadísticas secundarias y los datos corresponden a una selección de 44 países que son representativos de las disparidades intrarregionales de Europa y América Latina. ${ }^{23}$ Los países seleccionados

${ }^{22}$ L. Moreno, "Post-crisis and the Bronze Age of Welfare in Europe", en S. Segado y A. López (eds.), The Ailing Welfare State, Madrid, Thomson Reuters Aranzadi, 2016; Guillén, Luque y González, art. cit.

${ }^{23}$ Los países latinoamericanos considerados son: Argentina, Bolivia, Brasil, Chile, Colombia, Costa Rica, Guatemala, México, Perú, Uruguay, Ecuador, El Salvador, Honduras, Nicaragua, Paraguay, Panamá, República Dominicana y Venezuela. Mientras que los europeos son: España, Finlandia, Francia, Italia, Polonia, Portugal, Reino Unido, Rumania, Alemania, Austria, Bélgica, Bulgaria, Chipre, Croacia, Dinamarca, Eslovaquia, Eslovenia, Estonia, Grecia, Hungría, Letonia, Lituania, Malta, Países Bajos, República Checa y Suecia. 
dan cuenta de más de $85 \%$ de la población europea (excluyendo la parte europea de Rusia) y casi $96 \%$ de la población latinoamericana. Las fuentes de datos utilizadas fueron: el banco de datos del Banco Mundial, de la Oficina Estadística Europea (Eurostat), de la Comisión Económica para América Latina (Cepalstat) y de la Organización Internacional del Trabajo (ILOStat). Dado el propósito de comparar las trayectorias de estos países en el tiempo, el modelo de análisis comparado se aplica a aquellos seleccionados tras reunir información para cuatro periodos: 2000-2004, 2005-2008, 2009-2014 y 2015-2017.

En primer lugar, el cuadro 1 presenta la evolución de las desigualdades entre los países de cada región, entre regiones y a nivel total, así como sus cambios en el tiempo, con respecto a dos indicadores clave del bienestar: el PIB per cápita y la tasa de pobreza (con un umbral diario de USD 5.50, PPA). Con respecto al PIB per cápita, si bien tanto las regiones por separado como el agregado han mejorado, las diferencias entre Europa y América Latina persisten: incluso cuando la brecha se ha reducido levemente, el PIB promedio latinoamericano sigue siendo aproximadamente un tercio del europeo. El coeficiente de variación ha disminuido en Europa, pero se ha incrementado en el caso latinoamericano, ilustrando un crecimiento de la desigualdad entre países para América Latina en términos de ingreso promedio por habitante.

Con respecto a la incidencia de la pobreza, se observan mejoras tanto por región como para el agregado de países. La tendencia convergente se explica por el avance relativo de América Latina. De todas maneras, se advierten dos aspectos sustantivos. En primer término, como efecto de los diferentes niveles de desarrollo relativo, Europa presenta porcentajes de pobreza distintivamente bajos. En segundo lugar, la tendencia convergente soslaya la persistencia de fuertes diferencias entre países en cada región, más pronunciadas en los países europeos que en los latinoamericanos. 


\section{CuAdro 1}

Evolución de indicadores de bienestar: PIB per cápita y pobreza. América Latina y Europa (2000-2017)

\begin{tabular}{|c|c|c|c|c|c|}
\hline PIB per cápita (USD PPA) & $2000-2004$ & $2005-2008$ & 2009-2014 & $2015-2017$ & $\begin{array}{c}\text { Total } \\
2000-2017\end{array}$ \\
\hline \multicolumn{6}{|l|}{ Europa (26 países) } \\
\hline Promedio & 27171 & 31059 & 31020 & 33616 & 30717 \\
\hline Coeficiente de variación & $38.2 \%$ & $31.0 \%$ & $29.1 \%$ & $25.7 \%$ & $30.2 \%$ \\
\hline \multicolumn{6}{|l|}{ América Latina (18 paises) } \\
\hline Promedio & 9193 & 10681 & 12186 & 13178 & 11349 \\
\hline Coeficiente de variación & $42.6 \%$ & $44.0 \%$ & $43.7 \%$ & $44.1 \%$ & $42.4 \%$ \\
\hline \multicolumn{6}{|l|}{ Total (44 países) } \\
\hline Promedio & 19817 & 22722 & 23315 & 25536 & 22793 \\
\hline Coeficiente de variación & $61.5 \%$ & $56.6 \%$ & $51.9 \%$ & $49.5 \%$ & $54.1 \%$ \\
\hline \multicolumn{6}{|l|}{ Pobreza (5.5 USD PPA) } \\
\hline \multicolumn{6}{|l|}{ Europa (26 paises) } \\
\hline Promedio & 3.6 & 3.9 & 3.6 & 2.9 & 3.9 \\
\hline Coeficiente de variación & $149.9 \%$ & $186.1 \%$ & $165.2 \%$ & $178.0 \%$ & $158.9 \%$ \\
\hline \multicolumn{6}{|l|}{ América Latina (18 países) } \\
\hline Promedio & 45.6 & 36.6 & 28.6 & 21.6 & 34.0 \\
\hline Coeficiente de variación & $25.8 \%$ & $32.6 \%$ & $46.3 \%$ & $57.2 \%$ & $38.6 \%$ \\
\hline \multicolumn{6}{|l|}{ Total (44 países) } \\
\hline Promedio & $21 ., 9$ & 17.9 & 13.5 & 9.9 & 16.2 \\
\hline Coeficiente de variación & $103.9 \%$ & $105.5 \%$ & $115.1 \%$ & $125.9 \%$ & $109.4 \%$ \\
\hline
\end{tabular}

Fuente: elaboración propia con base en datos del Banco Mundial, Eurostat y Cepalstat para la muestra de países seleccionados.

Los resultados observados en materia de bienestar resultan esperables: los promedios regionales para los países considerados en cada caso presentan diferencias significativas que perduran en el tiempo. Ahora bien, ¿qué tan desiguales son estas sociedades en materia de distribución del ingreso? ¿Cuál ha sido su comportamiento en el periodo examinado? El cuadro 2 revela las diferencias estructurales entre las regiones objeto de estudio y un proceso de convergencia ocurrida en los últimos lustros en materia de desigualdad distributiva, evalua- 
da mediante el coeficiente de Gini. ${ }^{24} \mathrm{Si}$ bien los datos presentados son valores promedios, es evidente que el proceso de convergencia esconde una mayor caída relativa del coeficiente de Gini en los países de América Latina. ${ }^{25}$

Los datos también permiten examinar qué tanto convergieron los procesos de reducción/ampliación de la desigualdad en el interior de los países de cada región a través del coeficiente de variación de los índices de Gini. Se advierte que el proceso de convergencia presenta matices relevantes. Mientras la región latinoamericana registró una reducción de la desigualdad promedio, la desigualdad entre países no parece haber tenido cambios importantes. En Europa, en cambio, el leve incremento en el coeficiente de Gini después de la crisis de 2009 estuvo acompañado de una mayor heterogeneidad entre países. A nivel agregado, se observa evidencia en favor de la convergencia, que se expresa en la reducción del Gini promedio y del coeficiente de variación.

Un problema ampliamente abordado por la literatura del desarrollo es la relación entre los indicadores de bienestar y de desigualdad económica. Partiendo de la teoría del bienestar social, una mejora en el ingreso de los hogares o individuos podría ser considerada como evidencia de que el funcionamiento de la economía generó mejores condiciones materiales de vida para la población del país. Por ende,

${ }^{24} \mathrm{El}$ coeficiente de Gini es una medida de la desigualdad, generalmente empleado para evaluar la distribución del ingreso. Adopta valores entre 0 y 1 , siendo 0 la perfecta igualdad y 1 la perfecta desigualdad.

${ }^{25}$ Recientemente, algunas investigaciones analizan las posibles razones de la disminución de la desigualdad. J.P. Azevedo, G. Inchauste y V. Sanfelice, "Decomposing the Recent Inequality Decline in Latin America”, Policy Research Working Paper núm. 6715, World Bank, Washington, DC, 2013; A. de la Torre, J. Messina y J. Silva, "The Inequality Story in Latin America and the Caribbean: Searching for an Explanation", en L. Bértola y J. Williamson (eds.), Has Latin American Inequality Changed Direction? Looking Over the Long Run, Cham, Suiza, Springer Nature, 2017; N. Lustig, L. López-Calva y E. Ortiz-Juárez, "Deconstructing the Decline in Inequality in Latin America", en K. Basu y J. Stiglitz (eds.), Inequality and Growth: Patterns and Policy, Houndmills, Palgrave Macmillan, 2016. 
el ingreso podría emplearse como medida proxy del bienestar. Una mejora en los indicadores de distribución -expresada como reducción del coeficiente de Gini-, indicaría una menor desigualdad de las oportunidades de consumo, aportándole mayor "utilidad" a los individuos. ${ }^{26}$

\section{Cuadro 2}

Evolución del coeficiente de Gini. América Latina ${ }^{\mathrm{a}}$ y Europa $^{\mathrm{b}}$ (2000-2017)

$2000-2004 \quad 2005-2008 \quad 2009-2014 \quad 2015-2017 \quad 2000-2017$

\section{Europa (26 países)}

Promedio

0.287

0.299

0.299

0.302

0.297

Coeficiente de variación

$14.7 \%$

$14.6 \%$

$12.3 \%$

$13.6 \%$

$12.7 \%$

América Latina (18 países)

Promedio

0.527

0.508

0.480

0.467

0.496

Coeficiente de variación

$10.2 \%$

$9.0 \%$

$9.8 \%$

$10.5 \%$

$9.4 \%$

Total (44 países)

Promedio

$$
0.392
$$

0.387

0.373

0.370

0.378

Coeficiente de variación

$32.9 \%$

$29.3 \%$

$26.5 \%$

$25.3 \%$

$28.3 \%$

Brecha América Latina /

Europa

1.83

1.70

1.61

1.55

1.67

Fuente: elaboración propia con base en información del Banco Mundial, Eurostat y Cepalstat para muestra de países seleccionados.

a Coeficiente de Gini del ingreso per cápita familiar calculado en la distribución de personas.

b Coeficiente de Gini del ingreso equivalente (escala OCDE para normalización del tamaño del hogar) calculado en la distribución de personas.

${ }^{26}$ Más allá del impacto en el crecimiento y la pobreza, la desigualdad tiene un impacto directo y negativo en el bienestar social. Cfr. S. Yitzhaki, "Relative Deprivation and the Gini Coefficient", Quarterly Journal of Economics, 93, núm. 2 (1979), pp. 321-324. Asimismo, la desigualdad en el ingreso antes de impuestos puede conducir a una demanda de políticas redistributivas. Cf. T. Romer, "Individual Welfare, Majority Voting, and the Properties of a Linear Income Tax”, Journal of Public Economics 4, núm. 2 (1975), pp. 163-85. C. Shelton, "The Size and Composition of Government Expenditure", Journal of Public Economics 91, núms. 11-12 (2007), pp. 2230-2260. 
Dada la magnitud de las diferencias interregionales, es evidente que Europa y América Latina presentan diferentes modelos de desarrollo, regímenes de bienestar y rendimientos sociales. La evidencia empírica no es contundente en cuanto al sentido y la fuerza que tiene la relación entre estas dimensiones. Un óptimo de bienestar y equidad son metas de desarrollo esperables en la mayor parte de las sociedades del mundo, aunque no resulta legítimo inferir causalidad a partir de estas relaciones. ${ }^{27}$ En este marco, resulta de interés estudiar la relación entre la desigualdad y los procesos socioeconómicos e institucionales subyacentes para los países objeto aquí de estudio.

Con este propósito, se relaciona la media del coeficiente de Gini con indicadores de desarrollo económico (PIB per cápita) y de bienestar (tasa de pobreza absoluta). Al analizar la relación del Gini con el PIB per cápita y la pobreza, se advierte que los países con menor ingreso promedio son los más desiguales y que los más desiguales son también los más pobres (cuadro 3). En otros términos, una menor desigualdad por ingreso, medida a través del Gini, se encuentra positivamente correlacionada con el bienestar socioeconómico general, al menos para el promedio de los países considerados en este estudio durante el periodo 2000-2017.

\section{CuAdro 3}

Correlaciones para América Latina y Europa (2000-2017)

\begin{tabular}{lccc}
\hline & Gini & Pobreza & PIB per cápita \\
\hline Gini & 1 & & \\
Pobreza & $0.863^{* * *}$ & 1 & \\
PIB per cápita & $-0.832^{* * *}$ & $-0.833^{* * *}$ & 1 \\
\hline
\end{tabular}

Nota: significancia de los efectos: $* \mathrm{p}<0.05, * * \mathrm{p}<0.01, * * * \mathrm{p}<0.001$

Fuente: elaboración propia con base en datos del Banco Mundial, Eurostat y Cepalstat para muestra de países seleccionados.

${ }^{27}$ En otras palabras, la correlación entre variables no significa que exista una relación causal entre los dos eventos considerados. 
Un dato relevante es que esta relación no resulta tan marcada cuando el análisis se segmenta por regiones (cuadros 4 y 5). Al recalcular las correlaciones para cada región por separado, la fuerza de la relación entre las variables de bienestar y el Gini desciende considerablemente, aunque continúa siendo significativa. Este cambio da cuenta de la existencia de un tercer factor asociado con el Gini, el cual cabe vincular a las condiciones político-económicas de los sistemas nacionales de cada región. En este sentido, las diferencias señaladas se aprecian en las gráficas 1, 2 y 3 . Si bien se observan zonas de superposición, es evidente que se trata de dos conjuntos de países con matrices de desigualdad asociadas al bienestar, disímiles entre regiones, al tiempo que relativamente similares en el interior de cada región.

\section{Cuadro 4}

Correlaciones para países de América Latina (2000-2017)

\begin{tabular}{lccc}
\hline & Gini & Pobreza & PIB per cápita \\
\hline Gini & 1 & & \\
Pobreza & $0.550^{* * *}$ & 1 & \\
PIB per cápita & $-0.468^{* * *}$ & $-0.748^{* * *}$ & 1 \\
\hline
\end{tabular}

Nota: significancia de los efectos: $* \mathrm{p}<0.05, * * \mathrm{p}<0.01, * * * \mathrm{p}<0.001$

Fuente: elaboración propia con base en datos del Banco Mundial, Eurostat y Cepalstat para muestra de países seleccionados.

\section{CUADro 5}

Correlaciones para países de Europa (2000-2017)

\begin{tabular}{lccc}
\hline & Gini & Pobreza & PIB per cápita \\
\hline Gini & 1 & & \\
Pobreza & $0.509 * * *$ & 1 & \\
PIB per cápita & $-0.473^{* * *}$ & $-0.632 * * *$ & 1 \\
\hline
\end{tabular}

Nota: significancia de los efectos: $* \mathrm{p}<0.05, * * \mathrm{p}<0.01, * * * \mathrm{p}<0.001$

Fuente: elaboración propia con base en datos del Banco Mundial, Eurostat y Cepalstat para muestra de países seleccionados. 
GráfICA $1^{*}$

Correlación entre índice de Gini y PIB per cápita. Países de América Latina y Europa (2000-2017)

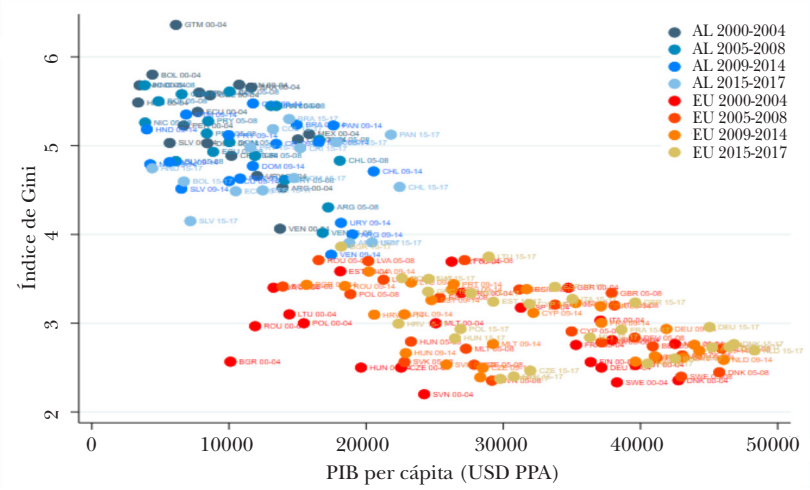

Fuente: elaboración propia con base en datos del Banco Mundial, Eurostat y Cepalstat para la muestra de países seleccionados.

\section{GrÁfICA 2}

Correlación entre el índice de Gini y la tasa de pobreza absoluta (USD 5.50 PPA diarios). Países de América Latina y Europa (2000-2017)

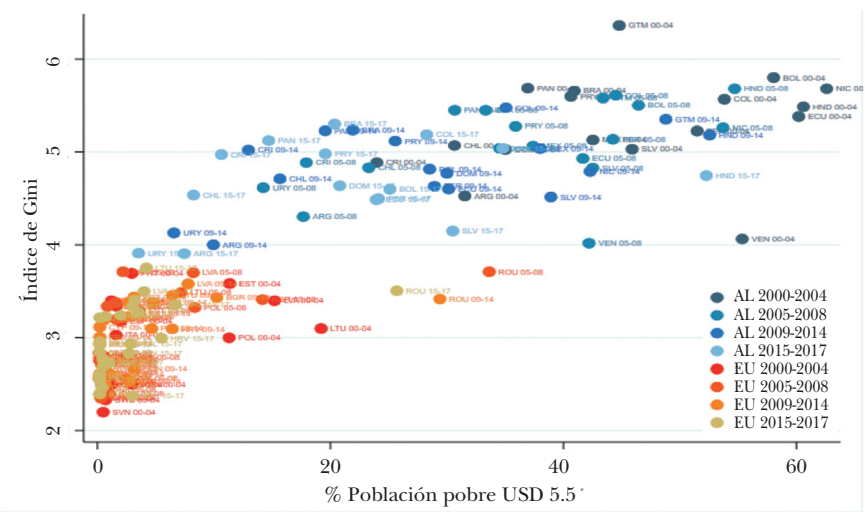

Fuente: elaboración propia con base en datos del Banco Mundial, Eurostat y Cepalstat para la muestra de países seleccionados.

* Gráficos a color en la versión digital y en blanco y negro en la impresa. 


\section{GrÁfICA 3}

Correlación entre tasa de pobreza absoluta (USD 5.50 PPA diarios) y PIB per cápita. Países de América Latina y Europa (2000-2017)

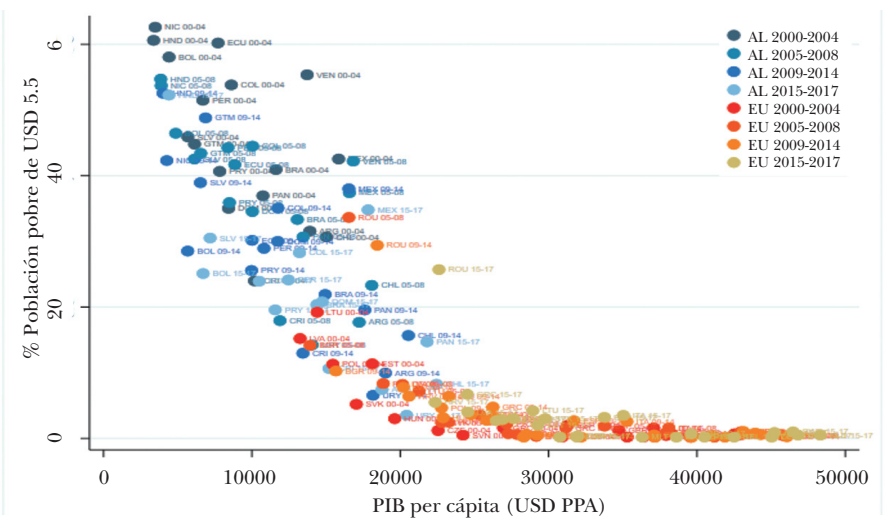

Fuente: elaboración propia con base en datos del Banco Mundial, Eurostat y Cepalstat para la muestra de países seleccionados.

RELACIÓN ENTRE LA DESIGUALDAD Y LAS FUENTES PRIMARIAS Y SECUNDARIAS DE DISTRIBUCIÓN DEL INGRESO

La reducción de la correlación entre los indicadores de bienestar y de desigualdad al controlar la región pone de manifiesto las diferencias estructurales entre las regiones comparadas. Ello motiva la pregunta sobre los procesos distributivos subyacentes asociados al comportamiento de la desigualdad. En este apartado se examina el papel distributivo del gasto público social como porcentaje del PIB y, por otra parte, el de la productividad del trabajo. Esta última puede ser interpretada como un proxy del valor del salario/remuneración. ${ }^{28}$

${ }^{28}$ La variable de productividad laboral empleada representa el volumen total de producción (РІв) producido por una unidad laboral (cantidad de personas ocupadas) durante un determinado periodo de tiempo. Este indicador de capacidades productivas brinda información general acerca de la eficiencia y la calidad del capital -tanto físico como humano- 
La evolución de ambos indicadores se presenta en las gráficas 4 y 5 , que dejan de manifiesto, una vez más, las desigualdades estructurales en el promedio de los países de ambas regiones. Las tendencias del gasto social y de la productividad media son positivas, aunque las brechas entre regiones continúan en el tiempo sin mostrar convergencia. Si se comparan estas evidencias con la evolución del Gini, se aprecia que los aumentos de productividad en Europa no redundaron en mayor bienestar ni en mayor igualdad, sino por el contrario, en una pérdida relativa de bienestar social. Inclusive, durante el último periodo se registra en esta región una caída de la participación del gasto social. En sentido contrario, América Latina registra en promedio un aumento constante tanto de productividad como en el gasto social, lo cual habría tenido un efecto positivo tanto en el bienestar como sobre la equidad.

Asimismo, si bien las diferencias entre regiones persisten, los comportamientos de ambos indicadores muestran ciertas divergencias. En particular, el gasto público social creció 31\% en América Latina, mientras que en Europa fue $5 \%$. En cuanto a la productividad media, el crecimiento también fue mayor en el caso latinoamericano (25\% versus $19 \%$ en Europa). Es decir que para el caso europeo la mejora en la productividad no estuvo acompañada de un aumento de la misma magnitud, o similar, en el gasto social. Este comportamiento permite conjeturar que la vigencia de instituciones más robustas en Europa garantiza un cierto patrón de distribución, con relativa flexibilidad, mientras que en América Latina las oscilaciones observadas darían cuenta de instituciones de bienestar menos desarrolladas (aunque en expansión) y, por lo tanto, de una mayor exposición de la matriz distributiva a las oscilaciones del mercado.

en el proceso productivo para un contexto económico y social dado. Véase Organización Internacional del Trabajo, OIT, "Productividad laboral", ILOSTAT Files, http://www.ilo.org/ilostat-files/Documents/description_ PRODY_SP.pdf 


\section{GrÁFICA 4}

Evolución del gasto público social de distintos niveles de gobierno. ${ }^{(1)}$ América Latina y Europa (2000-2017), en porcentaje del PIB

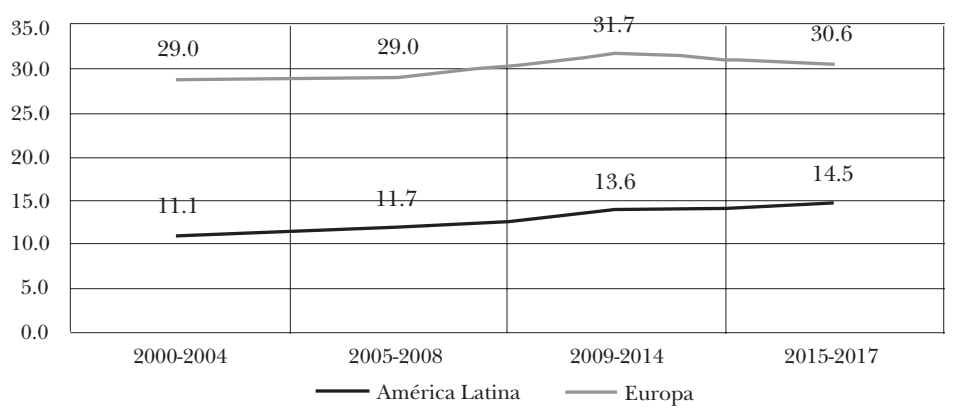

Notas: (1) Basado en la Clasificación de las Funciones de Gobierno (Cofog) de la OCDE.

Fuente: elaboración propia con base en información de en Eurostat y Cepalstat.

\section{GRÁFICA 5}

Evolución de la productividad promedio por trabajador. América Latina y Europa (2000-2017), en USD PPA 2011

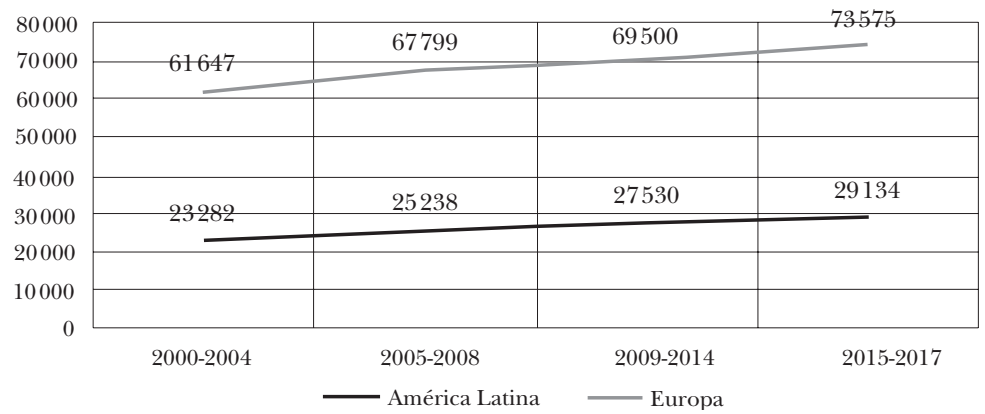

Fuente: elaboración propia con base en información de ILOSTAT.

En este marco, cabe preguntarse sobre el sentido y la fuerza de la correlación existente a nivel nacional entre el porcentaje del gasto social en el PIB y la productividad laboral (cuadros 6, 7 y 8). En todos los casos, ya sea en el agregado o 
en el desagregado por región, la relación entre gasto social y productividad promedio es positiva y significativa, aunque resulte más marcada en Europa que en América Latina. Ello refuerza la hipótesis de que el aumento de la productividad media incide en la capacidad de los países para generar excedentes orientados al gasto social y de que ello puede tener, a su vez, efectos positivos sobre la productividad. ${ }^{29}$ En cambio, la relación entre el Gini y el gasto social sólo es positiva y fuerte en el continente europeo, mientras que se diluye en el caso latinoamericano, lo que podría atribuirse a la existencia de otros factores -en especial, ligados al mercado laboral- que explican la desigualdad.

\section{Cuadro 6}

Correlaciones para América Latina y Europa (2000-2017)

\begin{tabular}{lccc}
\hline & Gini & Productividad & Gasto social \\
\hline Gini & 1 & & \\
Productividad & $-0.811^{* * *}$ & 1 & \\
Gasto social & $-0.827^{* * *}$ & $0.842^{* * *}$ & 1 \\
\hline
\end{tabular}

Nota: significancia de los efectos: $* \mathrm{p}<0.05, * * \mathrm{p}<0.01, * * * \mathrm{p}<0.001$

Fuente: elaboración propia con base en información del Banco Mundial, Eurostat y Cepalstat para muestra de países seleccionados.

\section{CUADRO 7}

Correlaciones para países de América Latina (2000-2017)

\begin{tabular}{lccc}
\hline & Gini & Productividad & Gasto social \\
\hline Gini & 1 & & \\
Productividad & $-0.475^{* * *}$ & 1 & \\
Gasto social & -0.192 & $0.344^{* *}$ & 1 \\
\hline
\end{tabular}

Nota: significancia de los efectos: $* \mathrm{p}<0.05, * * \mathrm{p}<0.01$, *** $\mathrm{p}<0.001$

Fuente: elaboración propia en base a Banco Mundial, Eurostat y Cepalstat para muestra de países seleccionados.

${ }^{29}$ R. Boyer, Is More Equality Possible in Latin America? (Working Paper núm. 67), International Research Network on Interdependent Inequalities in Latin America, Freie Universitat Berlin, 2014. 


\section{CuAdro 8}

Correlaciones para países de Europa (2000-2017)

\begin{tabular}{lccc}
\hline & Gini & Productividad & Gasto social \\
\hline Gini & 1 & & \\
Productividad & $-0.402^{* * *}$ & 1 & \\
Gasto social & $-0.517^{* * *}$ & $0.756^{* * *}$ & 1 \\
\hline
\end{tabular}

Nota: significancia de los efectos: $* \mathrm{p}<0.05$, ** $\mathrm{p}<0.01$, *** $\mathrm{p}<0.001$

Fuente: elaboración propia con base en información del Banco Mundial, Eurostat y Cepalstat para muestra de países seleccionados.

\section{Gráfica 6}

Correlación entre productividad promedio por trabajador e índice de Gini. Países de América Latina y Europa (2000-2017)

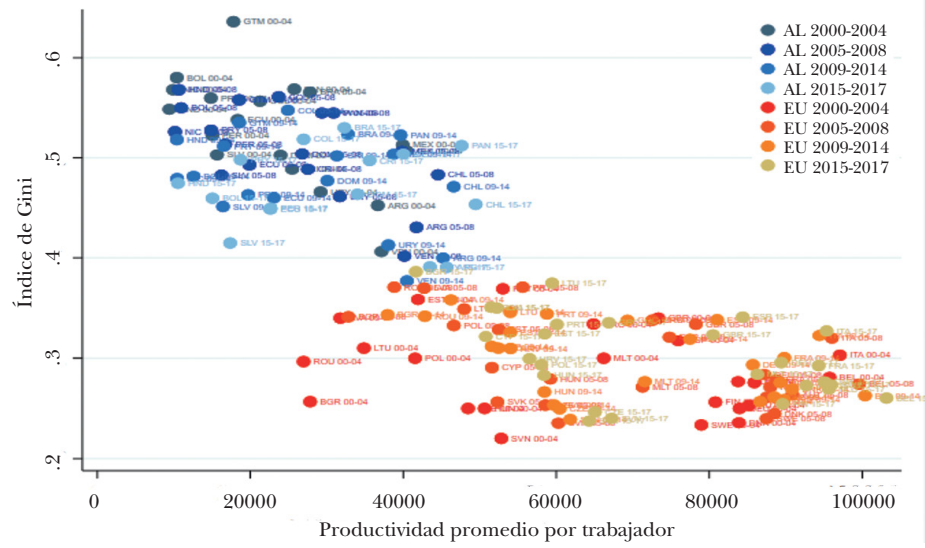

Fuente: elaboración propia con base en información del Banco Mundial, Eurostat y Cepalstat para muestra de países seleccionados. 


\section{GRÁFICA 7}

Correlación entre gasto social e índice de Gini. Países de América Latina y Europa (2000-2017)

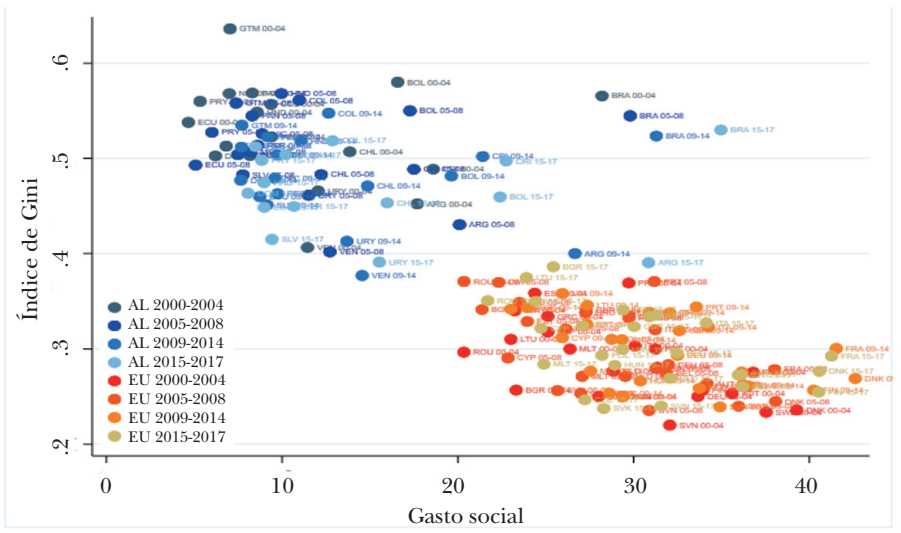

Fuente: elaboración propia con base en información del Banco Mundial, Eurostat y Cepalstat para muestra de países seleccionados.

\section{GrÁFICA 8}

Correlación entre productividad promedio por trabajador y gasto social. Países de América Latina y Europa (2000-2017)

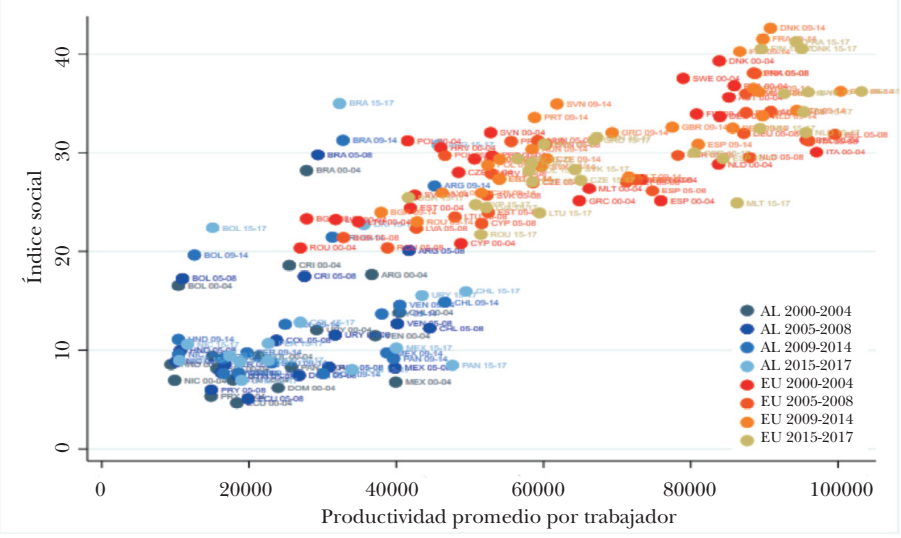

Fuente: elaboración propia con base en información del Banco Mundial, Eurostat y Cepalstat para muestra de países seleccionados. 
Los resultados alcanzados permiten formular la hipótesis de que, en contextos socioeconómicos con diferente grado de desarrollo económico, la distribución primaria de la renta es un factor clave para explicar tanto los niveles de bienestar como la distribución del ingreso, incluso en presencia de sistemas robustos de seguridad social encargados de garantizar un "mínimo" de ingresos. ${ }^{30} \mathrm{Si}$ bien estos sistemas pueden tener un impacto redistributivo importante, esto depende de su grado de madurez (sustentabilidad), cobertura y poder de transferencia, lo cual en última instancia también es función del desarrollo productivo y la distribución primaria del ingreso de cada país. ${ }^{31}$ Así, cabría aceptar que existen heterogeneidades estructurales e institucionales importantes entre los países de ambas regiones, tanto en materia productiva como en los sistemas de bienestar social. Ahora bien, los antecedentes tornan evidente que las regiones tienen un peso político-institucional propio, es decir, que las diferencias entre regiones resultan dominantes por sobre aquellas entre países de una misma región.

Con el fin de examinar este argumento de forma comparada, se ajustó un modelo factorial con las tres variables consideradas anteriormente: i) la desigualdad en la distribución del ingreso (índice de Gini), ii) la productividad laboral, y iii) el gasto social. La unidad de análisis son los periodos-naciones correspondientes a los 44 países estudiados, agrupados en cuatro ventanas temporales. El análisis factorial de componentes principales permite obtener patrones de diferenciación de los países-periodos. Este procedimiento permitió identificar dos factores subyacentes con dominancia sobre los procesos de (des)igualación económica entre las

${ }^{30}$ Organización Internacional del Trabajo, ort, Piso de protección social para una globalización equitativa e inclusiva, Ginebra, OIт, 2011.

${ }^{31}$ L. Moreno, La Europa asocial. Crisis y Estado del bienestar, Barcelona, Península, 2012. 
regiones. Ambos componentes explican casi el 95\% de la varianza total.

Por una parte, el vector "desarrollo con equidad" (eje horizontal) organiza los países según su nivel de desarrollo económico y sus implicaciones sobre la desigualdad: aquellos situados más a la derecha son simultáneamente más desarrollados e igualitarios; los situados hacia la izquierda son menos desarrollados y más inequitativos. Por otra parte, el vector "redistribución secundaria - inequidad" (eje vertical) organiza los países según la eficiencia compensatoria de su gasto social sobre la desigualdad: los que se sitúan más abajo tienen una relación más eficiente entre gasto social y equidad que los de más arriba, cuyo mayor gasto social no logra reducir la desigualdad. Al poner en correspondencia ambos factores se hace posible identificar diferentes "regímenes nacionales de desigualdad", ${ }^{32}$ tanto en el interior de Europa como de América Latina, además de describir sus principales desplazamientos en el tiempo. En efecto, siguiendo este modelo teórico-metodológico, es posible clasificar la posición factorial de las variables consideradas, así como la de los países-periodos tomados en el análisis.

La gráfica 9 da cuenta de la posición en el espacio factorial de las variables consideradas, lo cual permite identificar diferentes tipos posibles de trayectorias. En particular, tres principales: 1) reducción de la desigualdad debido a una mayor eficiencia redistributiva del gasto social y a una mejora distributiva primaria del ingreso derivada de un aumento de la productividad laboral; 2) estabilidad o incremento de la desigualdad con aumento de productividad pero con pérdida de eficiencia redistributiva del gasto social; 3) incremento de la desigualdad por empeoramiento de la distribución primaria dada una caída en la productividad laboral, así como un aumento ineficiente del gasto social. La pendiente de las trayectorias estará dada por la importancia relativa de los procesos identificados.

${ }^{32}$ En el sentido de Boyer, op. cit., 2014. 


\section{Gráfica 9}

Análisis de componente principal: componentes identificados y tipos de trayectorias para países de América Latina y Europa (2000-2017)

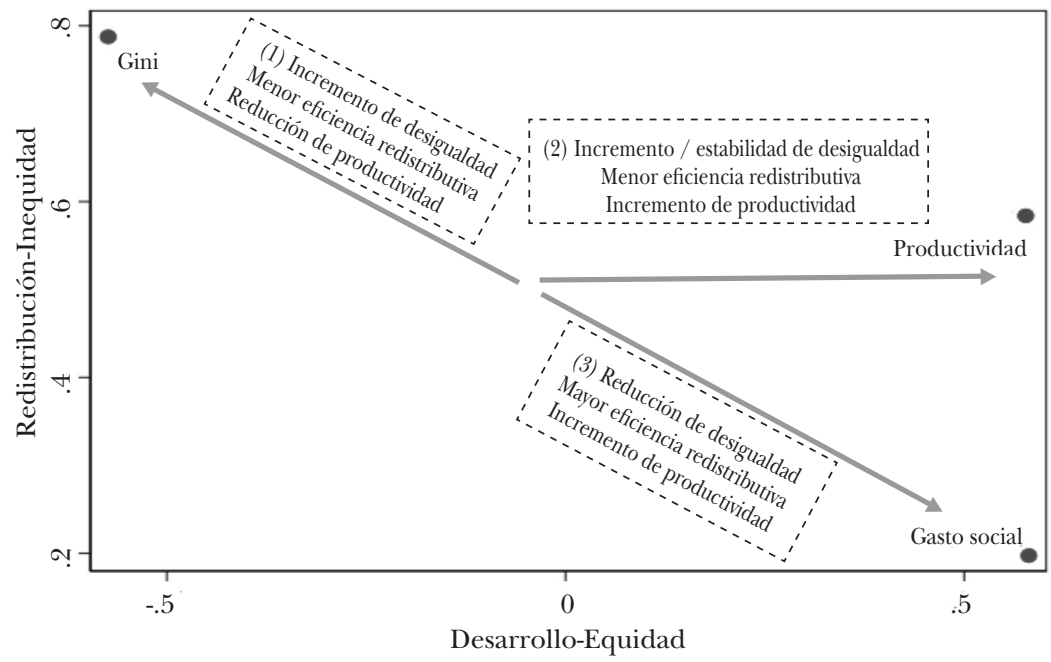

Fuente: elaboración propia con base en información del Banco Mundial, Eurostat y Cepalstat para muestra de países seleccionados.

La gráfica 10 distribuye a los países-periodos en torno a los ejes identificados y permite examinar, en primer lugar, cómo se distribuyen los casos en los cuadrantes delimitados por los ejes referidos y, en segundo lugar, cuál fue la trayectoria seguida por los países para los periodos considerados. Los ejes describen cuatro situaciones o tipos diferentes: (1) el cuadrante superior izquierdo reúne países con bajo desarrollo económico, elevada inequidad y baja eficiencia redistributiva del gasto social; (2) el cuadrante inferior derecho, países con alto desarrollo económico, baja desigualdad y eficiencia redistributiva del gasto social; (3) el cuadrante inferior izquierdo, países con bajo desarrollo económico, elevada desigualdad y eficiencia redistributiva compensatoria por el gasto social y (4) el cuadrante superior derecho, países con alto desarrollo econó- 
mico, moderada desigualdad y menor eficiencia redistributiva a través del gasto social.

\section{GrÁFICA 10}

Representación factorial de resultados del análisis de componente principal: scores para países de América Latina y Europa (2000-2017)

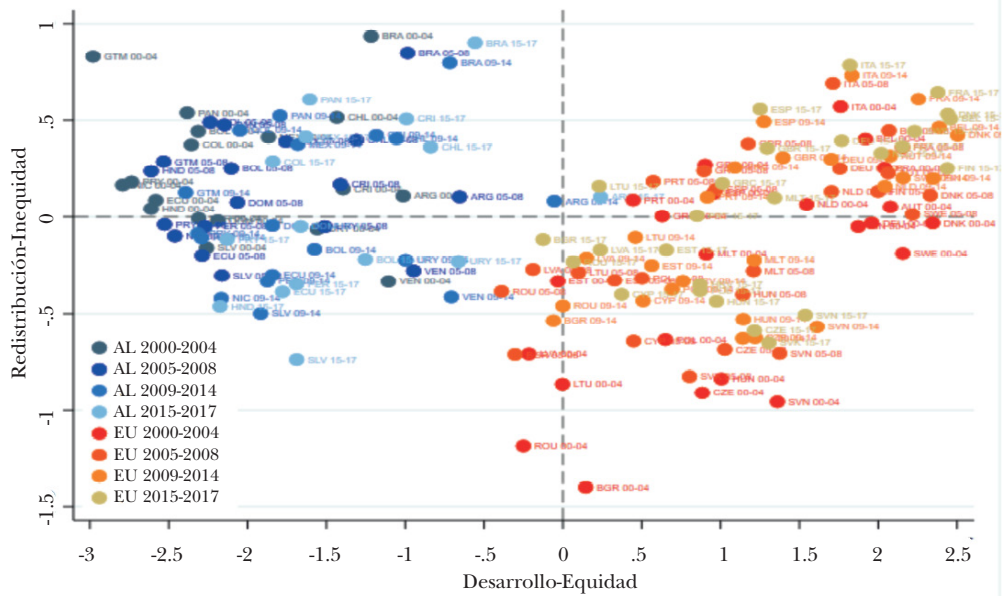

Fuentes: elaboración propia a partir de los resultados del análisis factorial de componentes principales (cuadro cruzado de factor 1 y factor 2), con base en datos del Banco Mundial, Eurostat y Cepalstat para muestra de países seleccionados.

A partir de esta matriz es fácil apreciar que, en forma independiente de los eventuales desplazamientos ocurridos durante el periodo 2000-2017, mientras los países-periodos de América Latina se concentran fundamentalmente en los cuadrantes izquierdos (menor desarrollo con mayor inequidad distributiva), los europeos se ubican de manera predominante en los cuadrantes derechos (mayor desarrollo con menor inequidad distributiva). Al mismo tiempo, los países-periodos de ambas regiones se dividen con relativa nitidez entre los cua- 
drantes superiores o inferiores de la gráfica según la efectividad del gasto social como factor asociado a una mayor o menor desigualdad (mayor desigualdad relativa con mayor gasto social versus menor desigualdad relativa con menor gasto social). En el marco de esta clasificación se registra la presencia de una serie de países-periodos europeos que destacan por su menor grado de desarrollo relativo pero alta eficiencia compensatoria por el gasto social (Bulgaria, Rumania, Lituania), los cuales sin embargo parecen desplazarse en forma ascendente. En el otro extremo destaca también la presencia de una serie de países-periodos latinoamericanos -fundamentalmente Brasil- con mayor desarrollo productivo relativo, pero con un elevado gasto social poco eficiente para reducir la desigualdad. En este caso, con un leve desplazamiento positivo hacia un mayor desarrollo con equidad.

Justamente, en materia de trayectorias, debido a que se trata de un espacio factorial, resulta más pertinente examinar su sentido que las distancias recorridas, aun cuando éstas puedan ofrecer información complementaria. Por otra parte, tal como se puede apreciar, si bien los países de América Latina y Europa recorrieron en tres lustros trayectorias disímiles, lo cual explica la convergencia distributiva ya discutida, no bastaron para alterar su distribución estructural en los cuadrantes anteriormente identificados.

Un primer examen intuitivo de las trayectorias permite confirmar que, más allá de situaciones particulares, la mayor parte de los países europeos registraron una trayectoria del tipo 2 (gráficas 11 y 12): relativo aumento o estabilidad en el nivel de desarrollo con aumento de productividad, pero sin reducción de la inequidad y, por lo tanto, con pérdida de eficiencia redistributiva del gasto social. Al respecto, cabe conjeturar que los efectos de la Gran Recesión y el tipo de recuperación que le sucedió imprimieron una marca específica a la inequidad distributiva. De manera simultánea, las políticas de austeridad implementadas habrían tenido por resultado la pérdida de eficiencia redistributiva del gasto social. Un proceso histórico que se ajusta bastante bien a lo que 
se denomina la "Edad de Bronce" del régimen de bienestar europeo. ${ }^{33}$

\section{GrÁfICA 11}

Recta de regresión sobre el factor desarrollo-equidad según periodo: scores para países de América Latina y Europa

(2000-2017)
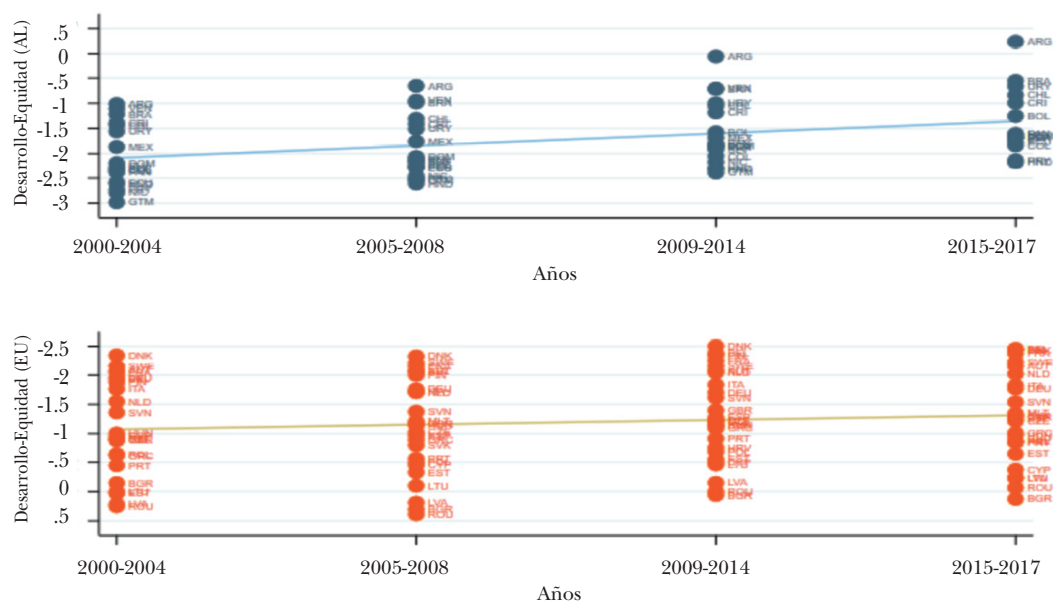

Fuentes: elaboración propia a partir de scores factoriales de la gráfica 10, con base en datos en datos del Banco Mundial, Eurostat y Cepalstat para muestra de países seleccionados.

Por el contrario, la mayor parte de los países latinoamericanos siguieron una trayectoria diferente que corresponde con el tipo 1: menor desigualdad por aumento de la productividad y mayor eficiencia redistributiva del gasto social y, por lo tanto, con efecto progresivo sobre la inequidad distributiva. Nuevamente, la pendiente de la trayectoria ofrece una idea de los logros en materia de reducción de la desigualdad. El modelo

${ }^{33}$ L. Moreno, 2017, op. cit. 
de crecimiento orientado al mercado interno y de políticas sociales expansivas (a menudo ligadas con pensiones asistenciales a la vejez y transferencias condicionadas), durante el boom de los precios de las commodities, explicaría el tipo de trayectoria observada.

\section{GRÁFICA 12}

Recta de regresión sobre el factor redistribución-inequidad según periodo: scores para países de América Latina y Europa (2000-2017)
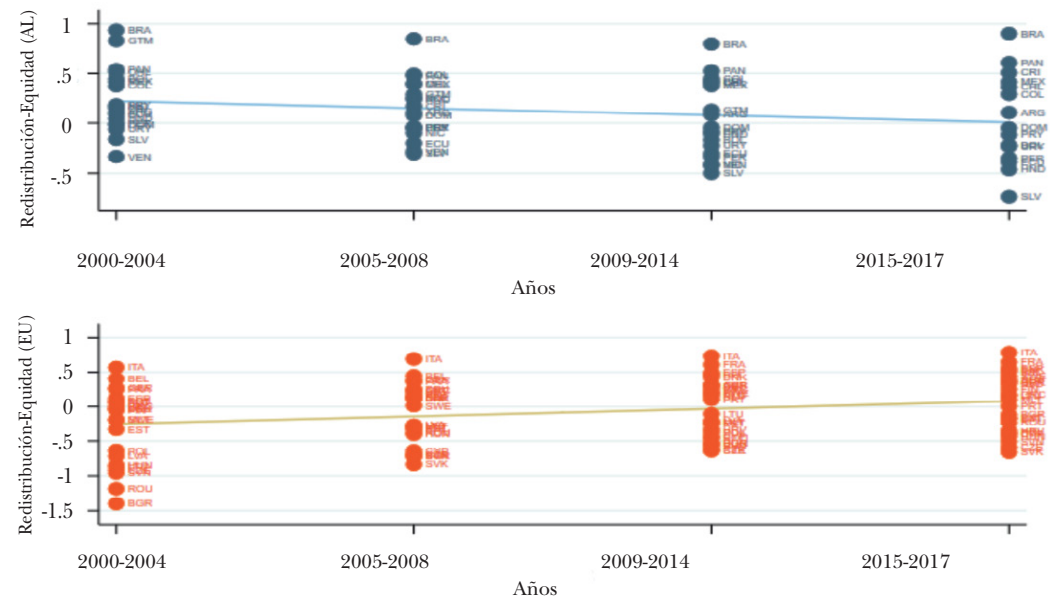

Fuentes: elaboración propia a partir de scores factoriales de la gráfica 10, con base en datos en datos del Banco Mundial, Eurostat y Cepalstat para muestra de países seleccionados.

A partir de los resultados obtenidos por este modelo de análisis, más allá de confirmarse que las desigualdades intrarregionales continúan siendo dominantes, se vuelve inteligible el proceso de convergencia que tuvo lugar en materia de distribución de la renta durante los primeros tres lustros del siglo XXI, en términos de los particulares procesos de distribución 
primaria y de distribución secundaria del ingreso que tuvieron lugar de manera casi unánime en los países de cada región. Ello se advierte en la distribución de los países-periodos en los ejes factoriales según la región de pertenencia. Es decir, el análisis desplegado permite constatar que las tendencias convergentes en materia de desigualdad resultan de una concatenación de procesos divergentes ligados al desarrollo productivo y a los diferentes modelos de política social implementados.

\section{Conclusiones}

En las últimas tres décadas, se han logrado importantes progresos en la superación de la pobreza absoluta a escala internacional. Sin embargo, la distribución del ingreso pone de manifiesto un escenario menos alentador. Distintos estudios han revelado que, si bien se verificaron procesos globales de convergencia en los niveles de renta, la inequidad interna en numerosos países ha tendido a incrementarse debido a la participación de los individuos y familias muy ricos en la distribución del ingreso. El impacto de estos cambios sobre los patrones de reproducción social de las desigualdades económicas, tanto en sentido como en magnitud, no ha sido suficientemente abordado por la literatura. Si bien existen estudios de casos nacionales o, incluso, análisis basados en comparaciones entre países de una misma región, son particularmente escasos los abordajes que comparan las matrices de desigualdad de ambas regiones.

En este artículo se llevó a cabo un análisis comparado de los cambios en la desigualdad entre países europeos y latinoamericanos durante los primeros lustros del siglo XXI. Asumiendo la presencia de matrices de bienestar e inequidad disímiles entre regiones-países, ¿cómo se explican esos diferentes niveles de bienestar, desigualdad económica y desarrollo y sus cambios recientes? Un primer acercamiento a este interrogante requirió describir la desigualdad en la dis- 
tribución de la renta nacional entre y en el interior de las regiones consideradas, así como sus cambios recientes en el tiempo, a partir de un modelo que vincula los coeficientes de Gini de cada país, en cuatro etapas diferentes del periodo 2000-2017, con las capacidades productivas de cada economía (distribución primaria del ingreso) y los esfuerzos redistributivos ejecutados por los gobiernos a través del gasto social (distribución secundaria del ingreso). A partir de este modelo, y siguiendo un análisis comparado entre países-periodos, se buscó identificar un patrón descriptivo estructurante de los diferentes modos en que los sistemas nacionales gestionan las desigualdades distributivas y sus cambios en el tiempo.

La caída en la relación entre los indicadores de bienestar y el coeficiente de Gini de los países presenta evidencia respecto a las diferencias estructurales existentes entre regiones, más allá de las correlaciones y diferencias que se presumen están presentes a nivel intrarregional. De acuerdo con la evidencia disponible, Europa y América Latina han reducido durante las últimas dos décadas del siglo Xxi sus brechas de desigualdad económica, pero mientras que la desigualdad distributiva se redujo en la mayor parte de los países de América Latina, un proceso inverso ocurrió al menos en la mayor parte de los países de Europa. Si bien ambas tendencias tuvieron excepciones, así como comportamientos nacionales diferentes en cada región, la evidencia analizada confirma estos procesos. En ambos casos, aunque con tendencias similares, la desigualdad intrarregional parece haber aumentado.

La caída observada en la correlación entre PIB per cápita e índice de Gini en ambas regiones motivó el análisis del papel que desempeñaron dos factores más directamente asociados al comportamiento de la desigualdad: el gasto social y la productividad. Del análisis de la relación existente entre gasto social y Gini se desprende que, a mayor gasto social, menor desigualdad en Europa; mientras que en América Latina esta relación se diluye y no es significativa. A la vez, se 
constató que la relación entre productividad y gasto social resulta positiva y significativa, en cualquier caso, aunque con más fuerza en América Latina. De esta forma, el análisis mostró que las capacidades de desarrollo productivo y los procesos de distribución secundaria del ingreso intervienen en la reproducción social de las desigualdades de manera diferente entre continentes.

El artículo abordó, a continuación, el modo en que los diferentes senderos de desigualdad transitados encuentran explicación en factores estructurales: por una parte, la productividad del trabajo (en clave a la heterogeneidad estructural, los modelos de regulación institucional y la segmentación laboral) y, por otro, en el modo en que el gasto social como porcentaje del PIB ha incidido en la reducción de la desigualdad (como expresión del nivel de cobertura y la capacidad redistributiva del Estado). En ese marco se realizó un análisis factorial-clasificatorio de los diferentes sistemas de desigualdad económica que caracterizan cada sistema nacional.

Dada una serie de indicadores sobre la productividad media del trabajo, el nivel de gasto social y sus efectos sobre el bienestar para cada país, se estableció un puente conceptual comparativo sobre la desigualdad distributiva del ingreso entre ambas regiones. El análisis dio cuenta de los procesos que explican los cambios en la desigualdad económica durante las últimas décadas. A partir de las tres variables consideradas (coeficiente de Gini, productividad laboral y gasto social), se identificaron dos componentes específicos para el análisis: "Redistribución e inequidad" y "Desarrollo y equidad". En general, los países latinoamericanos se desenvolvieron en torno al eje "Desarrollo y equidad", contando ya con ciertos niveles de redistribución. Los europeos, en cambio, se movieron más en torno al eje "Redistribución e inequidad" $y$, mientras algunos vieron disminuidos sus derechos sociales, otros los han mejorado.

Este trabajo aportó evidencias al debate político-académico acerca de los procesos de convergencia y divergencia en la desigualdad distributiva entre regiones. Una conclusión des- 
tacada es que la convergencia en los indicadores de desigualdad se explica por trayectorias nacionales divergentes. De esta forma, el artículo dio cuenta de factores estructurales que explicarían desigualdades distributivas intrarregionales que continúan siendo importantes. Más allá de los cambios producidos en cada región durante el siglo Xxi, las dinámicas interregionales no resultan convergentes en cuanto al desarrollo productivo, equidad y eficiencia del gasto social.

\section{REFERENCIAS BIBLIOGRÁFICAS}

AdLer, M. y K. Schmid, Factor Shares and Income Inequality: Empirical Evidence from Germany 2002-2008, IAW Discussion papers, núm. 82, mayo de 2012, http://www.iaw.edu/RePEc/iaw/pdf/ iaw_dp_82.pdf

Atkinson, A., The economics of inequality, Oxford, University Press, 1975.

Atrinson, A., Desigualdad: ¿Qué podemos hacer?, México, Fondo de Cultura Económica, 2016.

Atkinson, A., T. Piketty y E. Sáez, "Top Incomes in the Long Run of History", Journal of Economic Literature, 49 (1), 2011, pp. 3-71.

Azevedo, J.P., G. Inchauste y V. Sanfelice, "Decomposing the Recent Inequality Decline in Latin America”, Policy Research Working, Paper núm. 6715, World Bank, Washington, DC, 2013.

Barba Solano, C., "América Latina: regímenes de bienestar en transición durante los años noventa", Caderno CRH, 20 (50), 2007, pp. 197-211.

Barrientos, A. y D. Hulme, "Social protection for the poor and poorest: An introduction”, en A. Barrientos y D. Hulme (Eds.) Social protection for the poor and poorest. Palgrave Macmillan, Londres, 2008, pp. 3-24.

Boyer, R., Is More Equality Possible in Latin America? (Working Paper núm. 67), International Research Network on Interdependent Inequalities in Latin America, Freie Universitat Berlin, 2014. 
Daudey, E. y C. García-Peñalosa, "The personal and the factor distributions of income in a cross-section of countries". The Journal of Development Studies 43 (2), 2007, pp. 812-829.

Filgueira, F., "Modelos de desarrollo, matriz del Estado social y herramientas de las políticas sociales latinoamericanas", en S. Cecchini, F. Filgueira, R. Martínez y C. Rossel (eds.), Instrumentos de protección social. Caminos latinoamericanos hacia la universalización, Santiago de Chile, Cepal, 2015, pp. 49-84

Guillén, A. M., D. LuQue y S. González, "El modelo social eEuropeo: evolución y retos", en E. Del Pino y J. Rubio (Eds.), Los Estados del bienestar en la encrucijada. Políticas sociales en perspectiva comparada, Madrid, Tecnos, 2016.

GLyn, A., Functional Distribution and Inequality, Oxford, University Press, 2009.

Keeley, B., Income Inequality: The Gap Between Rich and Poor. OECD Insights, París, OECD, 2015.

Krugman, P., P. Wells y M. Olney, Fundamentos de Economía, Buenos Aires, Reverté, 2008.

Kuznets, S., "Economic Growth and Income Inequality". American Economic Review, 45, 1955, pp. 1-28.

Lustig, N., L. López-Calva y E. Ortiz-Juárez, "Deconstructing the Decline in Inequality in Latin America”, en K. Basu y J. Stiglitz (eds.), Inequality and Growth: Patterns and Policy, Houndmills, Palgrave Macmillan, 2016.

Milanovic, B., Desigualdad mundial. Un nuevo enfoque para la era de la globalización, México, FCE, 2016.

Molero-Simarro, R., "Growth and inequality revisited: the role of primary distribution of income. A new approach for understanding today's economic and social crises", Cambridge Journal of Economics, 41 (2), 2016, pp. 367-390.

Moreno, P. y B. Palier, "The Europeanization of Welfare: Paradigm shifts and social policy reforms", en P. Taylor-Gooby (ed.), Ideas and Welfare State Reform in Western Europe, Nueva York, Palgrave Macmillan, pp. 145-175, 2016.

Moreno, L., La Europa Asocial. Crisis y Estado del Bienestar, Barcelona, Península, 2012. 
Moreno, L., "Post-crisis and the Bronze Age of Welfare in Europe", en S. Segado y A. López (eds.), The Ailing Welfare State, Madrid, Thomson Reuters Aranzadi, 2016.

Organización para la Cooperación y el Desarrollo Económicos, ocDe, Perspectivas Económicas de América Latina y el Caribe 2014. Logística y Competitividad para el Desarrollo, OCDE, 2013.

Organización Internacional del Trabajo, oIT, Piso de protección social para una globalización equitativa e inclusiva, Ginebra, OCDE, 2011.

Organización Internacional del Trabajo, OIT, "Productividad laboral”, ILOSTAT Files, http:/ /www.ilo.org/ilostat-files/Documen ts/description_PRODY_SP.pdf

Organización Internacional del Trabajo, oIT, Wage-led Growth: An equitable strategy for economic recovery, Ginebra, Oficina Internacional del Trabajo, 2014.

Organización de las Naciones Unidas, ONU, Transformar nuestro mundo: Agenda 2030 para el desarrollo sostenible, Ginebra, ONU, 2013.

Ostry, J. y A. Berg, Inequality and Unsustainable Growth: Two Sides of the Same Coin? Washington DC., International Monetary Fund, 2011.

PiketTy, T., El capital en el siglo XXI, Madrid, FCE, 2014.

Romer, T., "Individual Welfare, Majority Voting, and the Properties of a Linear Income Tax", Journal of Public Economics 4 (2), 1975, pp. 163-85.

Shelton, C.,"The Size and Composition of Government Expenditure", Journal of Public Economics 91(11-12), 2007, pp. 2230-60.

Stiglitz, J., "Capital Market Liberalization, Economic Growth, and Instability", World Development, 28 (6), 2000, pp.1075-1086.

Therborn, G., La desigualdad mata, Madrid, Alianza Editorial, 2013. The World Bank, Globalization, Poverty, and Inequality since 1980, Oxford, University Press, 2005.

Torre, A. de la, J. Messina y J. Silva, "The Inequality Story in Latin America and the Caribbean: Searching for an Explanation”, en L. Bértola y J. Williamson (eds.), Has Latin American Inequality Changed Direction? Looking Over the Long Run, Cham, Suiza, Springer Nature, 2017. 
World Inequality Lab, Informe sobre la desigualdad global 2018, 2018, https://wir2018.wid.world

Yitzhaki, S., "Relative Deprivation and the Gini Coefficient", Quarterly Journal of Economics, 93 (2), 1979, pp. 321- 324.

Zalakain, H. y B. Barragué, Repensar las políticas sociales: predistribución e inversión social, Madrid, Editorial Grupo 5, 2017. 